bodies termed nucleoli and is surrounded by a double membrane pierced at intervals by pores.

Surrounding the nucleus is the cytoplasm in which are embedded various inclusions such as granules of secretion, globules of fat, and the thread-like or rod-like bodies known as mitochondria (dimensions $0.5-5 \mu$ by $0.3-0.7 \mu$ ). They are bounded by a double membrane, the inner layer of which is folded so as to produce a number of partitions and compartments. The mitochondria (of which there are about 400 in a liver cell) contain the enzymes responsible for oxidative phosphorylation and are the site of the production of high-energy compounds (such as adenosine triphosphate) in the cell.

The cytoplasm of the cell also contains a complex meshwork of canals and vesicles known as the endoplasmic reticulum or ergastoplasm with membranes about $5 \mathrm{~m} \mu$ thick separating the content of the tubules and vesicles from the general matrix of the cytoplasm. It is believed by some authorities that these tubules form a series of canals leading from the exterior of the cell to the nucleus. The surface of the tubules and vesicles in some areas is studded with small round electron-dense particles (diameter I0-20 $\mathrm{m} \mu$ ) known as ribosomes. They consist of about equal amounts of protein and ribonucleic acid (RNA) and are the organelles in which protein synthesis takes place in the cell. Ribosomes also occur free in the cytoplasm unattached to reticular membranes. Indeed, in certain types of cell (intestinal epithelium, tumour cells) the endoplasmic reticulum is scanty and most of the ribosomes are in the free state.

The general cell matrix or cell sap in which the endoplasmic reticulum and mitochondria are embedded contains a RNA of low molecular weight (soluble or transfer RNA) which in the process of protein synthesis is involved in the transfer of activated amino acids to the ribosomes.

When cells are disrupted in a suitable medium and are subjected to the process of differential centrifugation in order to separate the various subcellular components in accordance with their size and density, the remains of the endoplasmic reticulum appear in the form of microsomes (diameter 60-r $50 \mathrm{~m} \mu$ ) from which the ribosomes may be detached and separated by treatment with sodium deoxycholate.

\title{
Genetic determination of nutritional requirements
}

\section{By J. A. Roper, * Department of Genetics, University of Glasgow}

\section{Introduction}

A clear understanding of the genetic control of nutritional requirements came only after the fruitful union of biochemistry, genetics and microbiology. However, to present any account of the genetics of nutrition without a brief discussion of earlier fundamental work is to lose perspective.

The physician Garrod (1902, 1923), working on rare inherited metabolic disorders of man, such as alkaptonuria and cystinuria, was the first to show that genes act

\footnotetext{
* Present address: Department of Genetics, The University, Sheffield.
} 
through their influence on metabolic processes. Just how precise is this influence was demonstrated by studies of the genetic control of flower pigments (see review by Lawrence, 1950). The various anthocyanin pigments of flowering plants differ in their degree of methoxylation, hydroxylation and in the nature and position of the sugar residue. These factors, and the $\mathrm{pH}$ of the cell sap determine, in large measure, the colour of the flower. All these features of the anthocyanin pigments, and even the $\mathrm{pH}$ of the petal cell sap, are under simple genetic control.

A basic approach to the problems of genetics and biochemistry and hence, of course, to genetics and nutrition, was established by the studies of insect eye pigments (cf. Beadle, I945; Wagner \& Mitchell, r955). One great advance was to show that phenotypic correction of a genetically determined disorder might be achieved in a suitable environment. It was shown partly with the transplant technique by which imaginal discs from larvae of one genotype were transferred to larvae of a different genotype. Here the donor material was transferred to the new, exceedingly complex, environment of the host. Phenotypic correction was achieved when the host was able to supply, and the donor material accept, a metabolite capable of restoring the wild type phenotype. In one instance this principle was demonstrated with simple clarity. A red-eyed mutant strain of Drosophila melanogaster (vermilion; gene $v$ ) lacks a brown pigment which, in mixture with the red pigment, gives the wild type red-brown eye. Larvae of the vermilion strain, reared on a medium containing kynurenine, yield flies with the red-brown eye of the wild type. Such flies are, of course, still vermilion in genotype. Other red-eyed mutant strains of Drosophila, for instance cinnabar (gene $c n$, unlinked to $v$ ), remain red-eyed even when given kynurenine.

From such studies there gradually emerged a picture of a series of metabolic steps leading to the brown pigment which is missing in strains such as vermilion and cinnabar. Each step in the synthesis is gene controlled; mutation in the appropriate gene results specifically in failure of one step. Nutritional compensation, restoring the wild type phenotype, is possible when the environment supplies a diffusible nutrient which, in the reaction series, comes after the genetically blocked step. This finding explains why the vermilion strain responds to kynurenine whereas the phenotypically identical cinnabar strain does not.

The above studies pointed to a close association of genes and metabolism and so of genes and nutrition. But to present a broad picture based on work with higher organisms was likely to be difficult. Most such organisms are reared on complex media under non-aseptic conditions; further, most mutations identified in higher organisms are biochemically undefined. If such mutations do result from failure in some step of a metabolic process, then the missing metabolites are likely to be elusive. Even if the metabolites could be identified, nutritional compensation in a highly differentiated multicellular species might present considerable problems in terms of diffusibility of the added nutrients.

\section{Nutritional mutants of micro-organisms}

Beadle \& Tatum (I94I) were the first to show fully the potentialities of microorganisms for the study of biochemical genetics. They chose the mould Neurospora 
crassa. This species, apart from having a number of very convenient genetical features, offered considerable possibilities for biochemical genetics. It grows on a minimal medium of glucose, inorganic salts and biotin; from these it elaborates all the complex substances required for growth. On the assumption that these metabolic processes are genetically controlled there should be no difficulty in obtaining mutant strains unable to synthesize simple, readily diffusible substances. The fact that the organism has haploid nuclei throughout its vegetative cycle obviates the need for crossing to reveal recessive mutations. Also important was the fact that biochemical comparisons could be made between strains differing in only a single gene, which is impossible in most higher organisms.

From the wild type Neurospora, Beadle \& Tatum (I94I) derived mutant strains unable to grow on the minimal medium but able to grow on a complex medium. The mutations were, as regards the original minimal-medium environment, lethal. The spontaneous rate of occurrence of nutritional mutants is extremely low. The use of mutagenic agents and, later, the use of 'enriching' techniques (for references see Macdonald \& Pontecorvo, I953) made it a simple matter to isolate hundreds of nutritional mutants. Nutritional characterization and crossing of the mutants showed that, almost invariably, each mutant strain resulted from mutation in a single gene and each strain needed for growth a single substance not present in the minimal medium; mutation in a single gene produced, specifically, a single requirement. The range of nutritional types was impressive; it included strains requiring amino acids, purines, pyrimidines and vitamins. So far, there are few examples of mutants requiring larger types of molecules; it is uncertain how far this situation reflects problems of, for example, diffusion and how far it reflects shortcomings in the techniques for isolation of mutants.

A series of mutants might require, say, arginine. Crosses would show that such mutants fell into several different genetic classes; that is, mutation in any one of several genes resulted in arginine requirement. But this result could be explained in terms of the fact that arginine is synthesized in a number of steps. Cross-feeding experiments and tests of intermediates in arginine synthesis showed that each genetic group, in fact, required arginine or some different intermediate in arginine synthesis. As with the eye pigments of insects, it seemed that each step in a metabolic sequence was under genetic control. Mutation in a particular gene may result in failure of the step controlled by that gene. However, nutritional compensation for an otherwise lethal effect may be achieved by supplying any diffusible nutrient coming after the genetically blocked step. This type of study has now been undertaken in a wide range of microbial species (see review by Wagner \& Mitchell, 1955) and there can be no doubt that the Neurospora findings are, in essence, of universal application.

\section{The one gene-one primary product hypothesis}

Thus there were many instances, in higher organisms as well as in microorganisms, in which a single mutation appeared to result in the failure or modification of a single metabolic step. This finding led to the idea that many, perhaps all, 
genes have a single primary function. This idea is implicit in the writings of, among others, Garrod, Scott-Moncrieff, Wright, Haldane and Wheldale, and Grüneberg (1938) states it explicitly. Beadle (1945) took a bold step forward in elaborating his provocative one gene-one enzyme hypothesis. In essence Beadle's hypothesis stated that genes act through the determination of enzyme specificities and thus control synthetic and other metabolic processes. Each gene has a unique specificity and each enzyme will have its specificity set through one, and only one, gene. Mutation of a gene will result in failure of enzyme production or perhaps production of less enzyme or production of a modified enzyme. More recently Beadle (I95I) has revised the hypothesis to include within its scope not only enzymes but other biologically important macromolecules. Full substantiation of Beadle's hypothesis is difficult, if not impossible. For instance, proof of the absence of an enzyme, as opposed to the presence of an inhibited enzyme, is obviously at present impossible. However, there are a number of instances in which the enzyme of the mutant is not absent but is modified (Horowitz \& Fling, 1953; Fincham, 1957) and these lend strong support to Beadle's ideas.

Recent experiments with bacteria and bacteriophages (Avery, MacLeod \& McCarty, I944; Hershey \& Chase, I 952-3) strongly support the idea that the genetic material is deoxyribonucleic acid (DNA). A current view is that the DNA, probably through ribonucleic acid, determines the sequence of amino acids in enzymes and other proteins. The genetic information is assumed to be contained in the linear arrangement of purine and pyrimidine groups on the DNA structure. Stimulating suggestions about the genetic code and the way in which it might be translated into amino-acid sequences have already been made (Crick, 1958). It is possible, in microorganisms, to make an exceedingly fine linear analysis of the genetic material, perhaps to the extent of resolving distances involving one or a few nucleotide pairs (Benzer, 1955, 1957; Pontecorvo \& Roper, 1956). At the same time a detailed chemical analysis of protein is becoming a practical possibility. Combined studies, in which a fine genetic analysis is coupled with analysis of wild type and mutant proteins, may soon clarify fundamental aspects of gene action.

Most nutritional mutants can probably be considered in the light of the above explanation of the absence or modification of an enzyme concerned with synthesis of the required nutrient. However, some classes of mutants are not immediately and obviously explained in these terms and even the apparently simple may be deceptive. Sometimes we may have to consider alternatives, for example inhibitions due to genetic blocks in apparently unrelated reactions with consequent accumulation of intermediates; a further alternative may relate to matters of permeability. An example of a secondary effect is provided by the sulphonamide mutants of Neurospora (Emerson, I947; Zalokar, I948). The sulphonamide requirement appears to result from production of excess $p$-aminobenzoic acid since inhibition of $p$-aminobenzoicacid production results in loss of the sulphonamide requirement. Many instances of suppressor mutations are known. Such mutations 'reverse' the effects of other mutations, such as gene-determined nutritional requirements; the specificity of action of the suppressor varies in different situations. The modes of action of suppressors are 
unknown, but plausible guesses can be made. They may alter permeabilities, or affect $\mathrm{pH}$ in a way favourable for a modified or inhibited enzyme to function, or act through the loss of an enzyme inhibitor, or perhaps through the opening of an alternative route of synthesis. Mutations dependent for their expression on a particular $\mathrm{pH}$ or temperature have also been studied; some of them may well result from enzyme modification. Partial mutants which show limited growth without added nutrients and full growth with them may result from impaired or partially inhibited enzymes. Although the instances just mentioned may be difficult to interpret in our present state of knowledge, it seems likely that they all fall within the scope of the one gene-one primary product idea.

\section{Recent trends in the study of nutritional mutants}

So far I have attempted to give a broad and fairly general picture. It may now be worth while to consider a few points of special interest in the present context.

Nutritional mutants have been used to elucidate metabolic pathways and for the identification and isolation of accumulated, sometimes unsuspected, intermediates (see review by Wagner \& Mitchell, 1955). Such mutants have also revealed instances of competitive inhibitions between normal metabolites. For instance, certain arginine-requiring mutants of Neurospora crassa (Srb \& Horowitz, 1944; Doermann, I944) and Aspergillus nidulans (Pontecorvo, I953) are competitively inhibited by added lysine and certain lysine-requiring mutants are competitively inhibited by exogenous arginine. Neither arginine nor lysine inhibits the wild type strains. Pontecorvo (r950). has suggested that such inhibitions may result from competition at a special site in the cell, perhaps at a membrane.

Comparisons of nutritional responses in various species have revealed interesting differences (Pontecorvo, 1953). For instance, the proline- and ornithine-requiring mutants of Aspergillus, in distinction to those of Neurospora, do not respond to citrulline though in both species these mutants respond to arginine. This distinction could reflect differences in permeability or in metabolic pathway. Interspecific comparisons may gradually lead us to some ideas of biochemical divergences and evolution.

One final point is well worth inclusion. Pontecorvo (1950) proposed a working hypothesis aimed at a rational approach to the question of whether genes are distributed along the chromosomes at random, regardless of their functions, or whether the linkage arrangement of the genes reflects, in some instances, a biochemical relationship. Pontecorvo's hypothesis can be stated briefly as follows. Close linkage might be expected between some of the genes acting on any one series of biochemical reactions when the intermediates are labile, non-diffusible, or are produced in very low concentration. This provocative hypothesis prompted a number of investigations in Aspergillus (Roper, 1950, 1953; Pritchard, 1955) but these studies cast light on intragenic rather than intergenic organization. It remained for Demerec \& Hartman (r 956) and Hartman (r 956), using Salmonella typhimurium, to demonstrate clear-cut instances of the type postulated by Pontecorvo. For instance, tryptophan 
biosynthesis in Salmonella follows the path
A
B
C
$\mathrm{D}$
$\rightarrow$ anthranilic acid $\rightarrow$ compound ' $\mathrm{B}$ ' $\rightarrow$ indole $\rightarrow$ tryptophan.

Genes $A, B, C$ and $D$ control the steps in this series of reactions. These genes are, in fact, closely linked and in the order ABCD on the bacterial 'chromosome'. The gene sequence reflects the biochemical sequence and shows submicroscopic organization of metabolic processes and, therefore, of processes of intracellular nutrition.

\section{Conclusion}

The application of genetical ideas and techniques to the nutrition of micro-organisms has brought a clearer understanding of the relationship of genotype and nutritional requirement. It has opened a new approach and a new way of thought to a large aspect of genotype-environment interaction. Further advances will lead to practical applications in fields as separated as those of antibiotic production and psychiatry (Roper, 1958a). On the academic side there remain many problems. Some of them will be concerned largely with the reaction of organism and environment and, in any event, that is an essential preliminary. At a different level, there are problems of the biochemical relationships of structures and entities within the cell or organism. Some ways of attack on a few of these problems are already suggested by studies such as those on Salmonella, by work on heterokaryosis in filamentous fungi, by investigations (Roper, 1958b) which suggest that localized cytoplasmic changes can influence nutritional requirements and, of course, by the work on biochemical activities of isolated cell constituents. At this level the boundaries of genetics, biochemistry and nutrition are blurred, but the problems to be formulated and solved in fact relate to intracellular aspects of nutrition. Attack on these problems must surely be one important aim of genetical-nutritional studies.

\section{REFERENCES}

Avery, O. T., MacLeod, C. M. \& McCarty, M. (1944). F. exp. Med. 79, 137 .

Beadle, G. W. (1945). Chem. Rev. 37, 15 .

Beadle, G. W. (195I). In Genetics in the 2oth Century. (L. C. Dunn, editor.) New York: Macmillan.

Beadle, G. W. \& Tatum, E. L. (1941). Proc. nat. Acad. Sci., Wash., 27, 499.

Benzer, S. (1955). Proc. nat. Acad. Sci., Wash., 4I, 344.

Benzer, S. (1957). In The Chemical Basis of Heredity. (W. D. McElroy and B. Glass, editors.) Baltimore: Johns Hopkins Press.

Crick, F. H. C. (1958). Sym. Soc. exp. Biol. 12, ${ }_{3} 8$.

Demerec, M. \& Hartman, Z. (1956). Publ. Carneg. Instn, 612, 5.

Doermann, A. H. (1944). Arch. Biochem. 5, 373.

Emerson, S. (1947). F. Bact. 54, 195.

Fincham, J. R. S. (1957). Biochem. F. 65, 721.

Garrod, A. E. (1902). Lancet, ii, I6I6.

Garrod, A. E. (1923). Inborn Errors of Metabolism, 2nd ed. Oxford: University Press.

Grüneberg, H. (1938). Proc. roy. Soc. B, 125, 123 .

Hartman, P. E. (1956). Publ. Carneg. Instn, 612, 36.

Hershey, A. D. \& Chase, M. (1952-3). F. gen. Physiol. 36, 39.

Horowitz, N. H. \& Fling, M. (1953). Genetics, 38, 360.

Lawrence, W. J. C. (1950). Biochem. Soc. Symp. 4+3.

Macdonald, K. D. \& Pontecorvo, G. (1953). Advanc. Genet. 5, 159.

Pontecorvo, G. (1950). Biochem. Soc. Symp. 4, 40.

Pontecorvo, G. (1953). Advanc. Genet. 5, I41.

Pontecorvo, G. \& Roper, J. A. (1956). Nature, Lond., x78, 83. 
Pritchard, R. H. (1955). Heredity, 9, 343.

Roper, J. A. (1950). Nature, Lond., 166, 956.

Roper, J. A. (1953). Advanc. Genet. 5, 208.

Roper, J. A. (1958a). In Topics in Psychiatry. (T. Ferguson Rodger, R. M. Mowbray and J. R. Roy, editors.) London: Cassell.

Roper, J. A. (1958b). Cold Spr. Harb. Symp. quant. Biol. 23, 14I.

Srb, A. M. \& Horowitz, N. H. (1944). F. biol. Chem. 154, 129.

Wagner, R. P. \& Mitchell, H. K. (1955). Genetics and Metabolism. London: Chapman \& Hall.

Zalokar, M. (1948). Proc. nat. Acad. Sci., Wash., 34, 32.

\section{The nutrition of animal cells in vitro}

\section{By J. Paul, H.E.R.T. Tissue Culture Laboratory, Department of Biochemistry, University of Glasgow}

The nutritional requirements of isolated animal cells have been the subject of numerous studies since tissue-culture techniques were first developed in the early years of this century. Earlier investigators were handicapped by the apparent necessity of using complex biological media, such as plasma and embryo extract. The first material contribution to cell nutrition was made by Albert Fischer and his colleagues (Fischer, Astrup, Ehrensvard \& Oehlenschlager, 1948) who dialysed the plasma and embryo extract before use and made a systematic investigation of the substances required in addition to the non-dialysable material. They were able to demonstrate that the requirements for small molecules could be met by supplying a mixture of amino acids, vitamins and a few other substances. Further advances had to await the development of new methods of cell cultivation in the last decade. The most important of these have been: (I) the demonstration that the plasma clot can nearly always be completely dispensed with and that cells will grow satisfactorily directly on glass, (2) the development of replicate methods of cell culture by treatment of tissue or cultures with proteolytic enzymes to obtain cell suspensions, (3) the isolation of pure strains of cells derived from single cells and (4) the development of synthetic media which will support cell growth when supplemented with only a very small amount of dialysed serum. Very recently it has been found possible to dispense with serum in a few instances.

These advances made it possible to investigate the minimal nutritional requirements of many animal cells. Three general criteria have been used for this purpose. Most workers have relied on rapid cell proliferation, defining essential nutrients as those necessary for the maintenance of rapid cell growth. Morgan's group (Morton, Pasieka \& Morgan, I956), on the other hand, has used cell survival, and has defined essential components in terms of their ability to maintain the viability of previously starved cells. In general the results obtained by the two groups are in agreement. Some other workers have pointed out that even rapidly growing cell strains are not using all their synthetic or functional capacities and have suggested that nutritional requirements should be defined in terms of the ability to maintain full function. Of the three criteria, the ability to maintain rapid growth has been most widely used 